\title{
Thromboelastography Use in the Perioperative Transfusion Management of a Patient with Hemophilia A Undergoing Liver Transplantation
}

\author{
Alejandro Mejia ${ }^{1 *}$, Maria L. Mendoza ${ }^{1}$, Catalina Mejia ${ }^{1}$, Gong W. Lee ${ }^{2}$ \\ ${ }^{1}$ Transplant Surgery, The Liver Institute at Methodist Dallas, Dallas, USA \\ ${ }^{2}$ Methodist Dallas Medical Center, Dallas, USA \\ Email: *alejandromejia@mhd.com
}

Received September 29, 2012; revised October 30, 2012; accepted November 8, 2012

\begin{abstract}
The thrombelastogram is a method used to monitor clotting dynamics. Thrombelastography (TE) has been used to guide therapy of coagulation disorders mostly in cardiac surgery but also in liver surgery. TE is a useful tool for perioperative management of patients at risk for coagulopathy. There are several reports describing the use of the thrombelastogram in patients undergoing orthotopic liver transplantation (OLT), but only few cases include patients with both liver disease and inherited bleeding disorders. We describe the use of TE in a patient with hemophilia A and advanced cirrhosis undergoing OLT.
\end{abstract}

Keywords: Hemophilia; Liver Transplantation; Thromboelastography

\section{Introduction}

The thrombelastogram is a method used to monitor clotting dynamics. Thrombelastography (TE) has been used to guide therapy of coagulation disorders mostly in cardiac surgery but also in liver surgery. TE is a useful tool for perioperative management of patients at risk for coagulopathy. There are several reports describing the use of the thrombelastogram in patients undergoing orthotopic liver transplantation (OLT), but only few cases include patients with both liver disease and inherited bleeding disorders [1] .We describe the use of TE in a patient with hemophilia A and advanced cirrhosis undergoing OLT.

\section{Case}

A 45-year-old Hispanic male with history of hemophilia A and cirrhosis secondary to hepatitis C infection was admitted for OLT. His diagnosis of hemophilia A was known since age of five and his family history was significant for hemophilia in two brothers and one niece.

As a complication of cirrhosis he developed ascites, esophageal varices, coagulopathy and encephalopathy. He also developed pulmonary hypertension and was placed on an Epoprostenol drip for a total of 6 months prior to the transplant.

"Corresponding author.
Preceding the transplant event, the patient was admitted because of profound blood loss through an anal fissure. His initial workup showed a hemoglobin of $3.7 \mathrm{~g} / \mathrm{dl}$, platelet count of 46,000 u/L, prothrombin time (PT) of 22.1 seconds, international normalized ratio (INR) 1.9, partial thromboplastin time (PTT) of 58 seconds and a factor VIII level activity of 5\%. Hematological management included: serial factor VIII infusions, packed red blood cells, platelets and cryoprecipitate transfusions.

Ten days after admission a liver was offered. His model for end stage liver disease (MELD) score was 22. At this time, the patient's factor VIII level activity was $32 \%$, PT was 16.3 seconds, INR was 1.3, hemoglobin 9.2 $\mathrm{g} / \mathrm{dl}$, platelet count was $20,000 \mathrm{u} / \mathrm{L}$ and fibrinogen level was $193 \mathrm{mg} / \mathrm{dl}$. The pretransplant hematology protocol consisted of: factor VIII administration of 50 units $/ \mathrm{kg}$ one hour prior to skin incision, transfusion of 10 units of cryoprecipitate, one single donor platelet (SDP) and two units of fresh frozen plasma (FFP). Factor VIII serial infusions were continued for 6 days.

An OLT was performed with a liver from a 27-yearold donor. The operation was uncomplicated and a piggyback technique was used. The cold ischemic time was 5 hours and 26 minutes and the warm ischemic time was $35 \mathrm{~min}$.

During the operation, a thrombelastogram was obtained immediately before transplant (Initial); immediately after completing the native hepatectomy (1st an- 
hepatic); immediately prior to unclamping the new allograft (2nd anhepatic); 30 minutes after reperfusion (Reperfusion) and upon arrival to the intensive care unit (ICU). A follow up thrombelastogram was done 6 days post transplant prior to discharge. Intraoperative TE was obtained with the TEG $^{\circledR}$ system (Haemoscope-Haemonetics Corp., Braintree, MA, 2008) .The software used was 4.2.3 TEG 5000 Thrombelastograph Hemostasis System (Braintree, MA). A Rapid TEG ${ }^{\circledR}$ was used in the operating room. This test is performed with whole blood and gives a faster reading. A Basic TEG ${ }^{\circledR}$ was used during the ICU period. This test uses heparinase and can either demonstrate the presence of heparin or monitor coagulation in the presence of full heparinization.

The initial Rapid TEG ${ }^{\circledR}$, perioperative and postoperative period TEG ${ }^{\circledR}$ values are shown (Figures 1-5).

The total blood products transfused during surgery in- cluded: 8 units of PRBC, 1 unit of cell saver, 16 units of FFP, 3 units of SDP and 20 units of cryoprecipitate. Desmopressin was not used and the estimated blood loss during surgery was 3 liters. During the ICU period he received 3 units of PRBC and 3 units of SDP.

At the time of discharge, 6 days post OLT; the TEG ${ }^{\circledR}$ values demonstrated an MA value of 41.8, G of 3.6, and alpha angle of 47.7 . Despite altered TEG $^{\circledR}$ values reflecting a hypocoagulable state, the patient did not experience clinically significant bleeding in the postoperative period. More important, despite the factor VIII infusion, the $\mathrm{TEG}^{\circledR}$ demonstrated no hypercoagulability. A summary of all TEG ${ }^{\circledR}$ findings is included in Table $\mathbf{1}$.

The allograft function remained stable and inmunosuppression was started with standard triple regimen. He was weaned off Epoprostenol and is doing well five years post transplant.

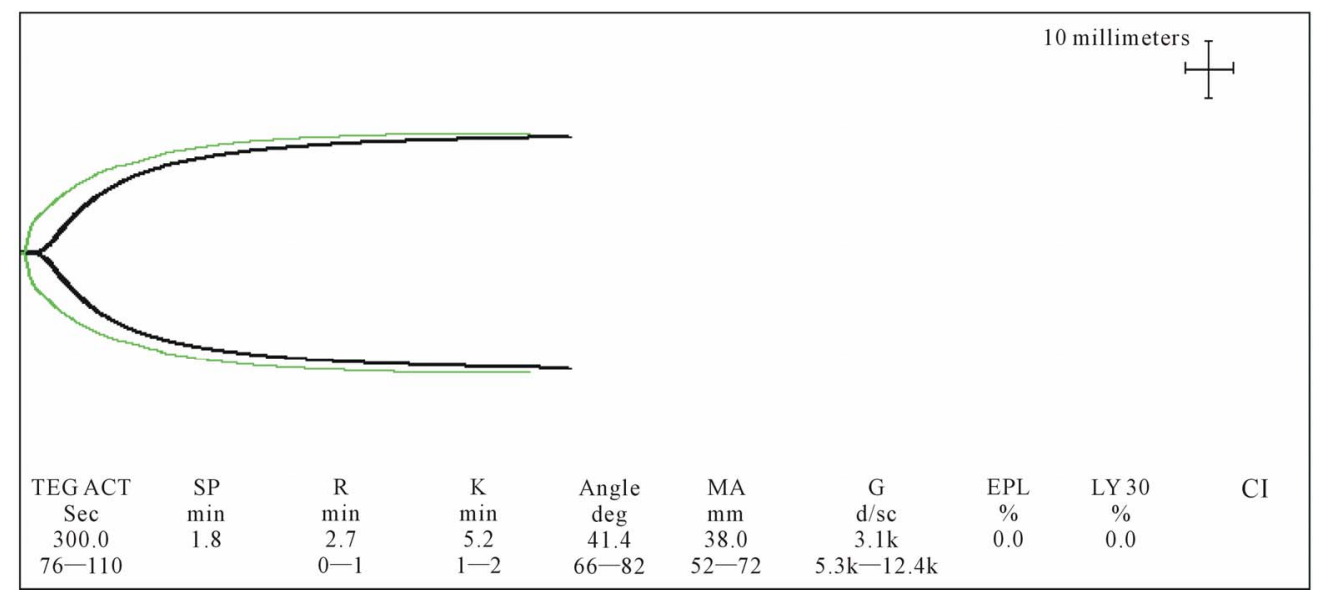

Figure 1. Initial Rapid TEG ${ }^{\circledR}$ Rapid TEG ${ }^{\circledR}$ : values corresponded to an activated clotting time (ACT) of 300 seconds, which reversed to 105 seconds with heparinase, indicating the presence of endogenous heparinoid. The alpha angle of 41.4 degrees reflected low platelet count and low fibrinogen level. The MA (maximal amplitude) of $38 \mathrm{~mm}$ was 5 SD below the mean, due to low platelet count and to Epoprostenol infusion side effect of inhibiting platelet aggregation. The clot strength (G) of $3.1 \mathrm{k}$ (3.2 SD below the mean) indicated poor interaction between fibrinogen, platelets and clotting factors.

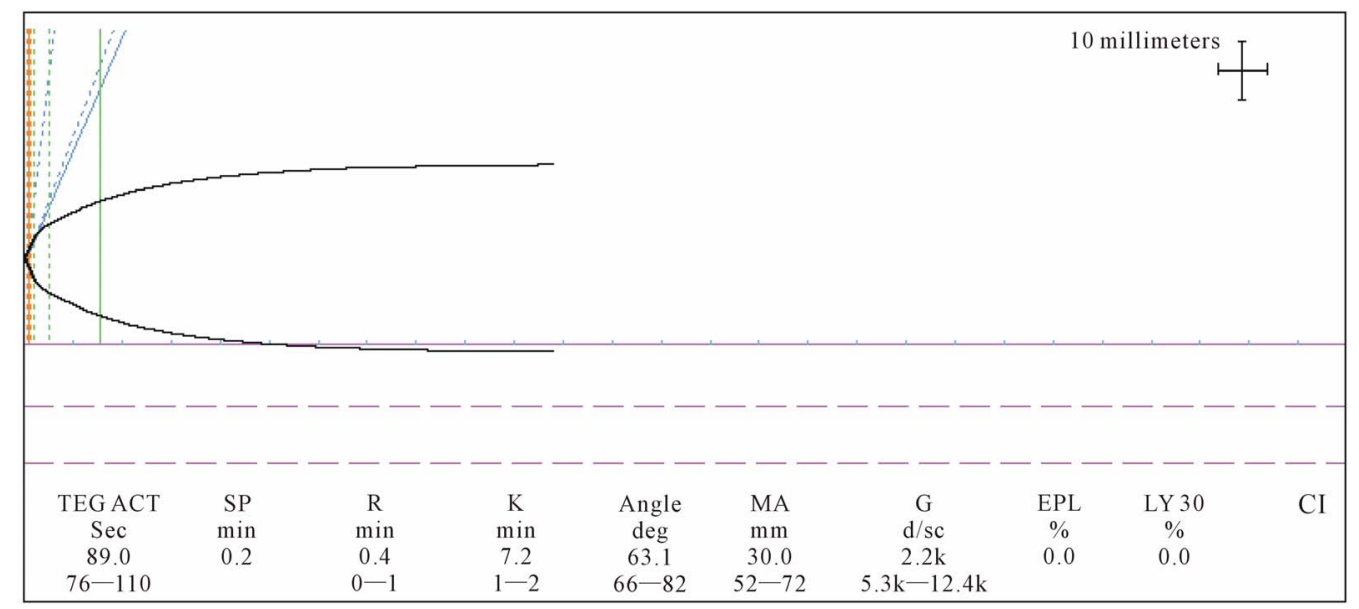

Figure 2. First Anhepatic phase Rapid TEG ${ }^{\circledR}$. The alpha angle was of 63.1 degrees (2.7 SD below the mean). The MA and G values were below normal. At this point the patient received 1 SDP based on the MA and the platelet count of 31,000 u/L. 


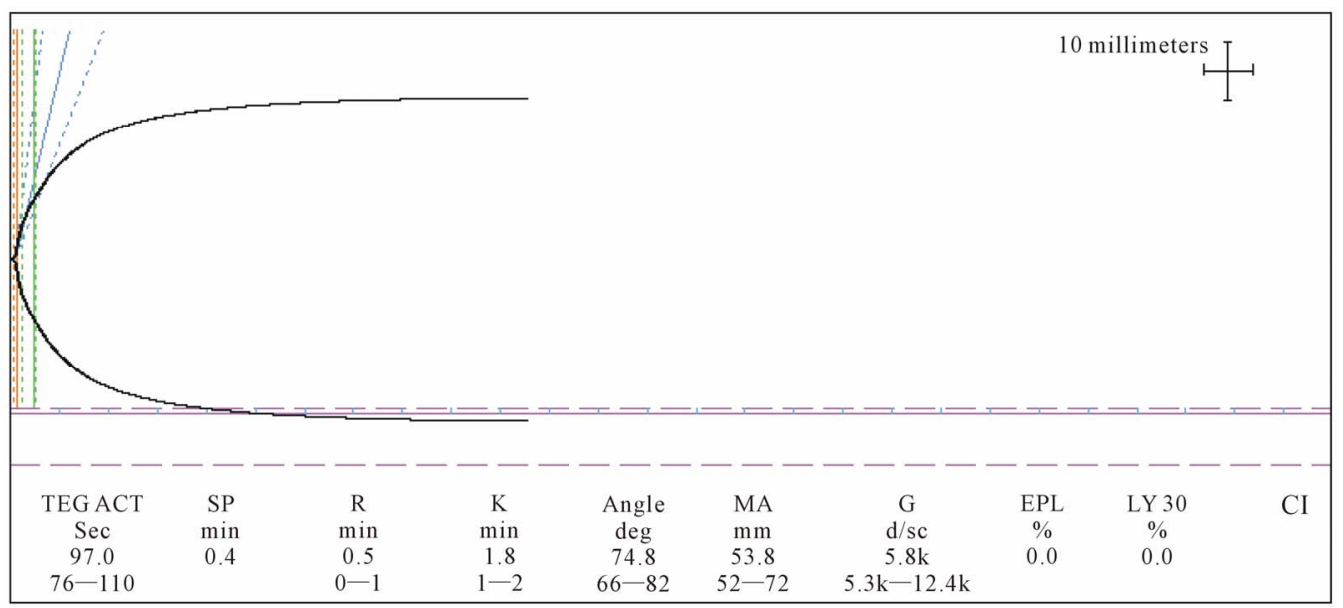

Figure 3. Second Anhepatic Rapid TEG ${ }^{\circledR}$ The values, showed an ACT of 97 seconds indicating good clotting factor function. The alpha angle was within normal range. The MA of 53.8 and $G$ of 5.8 were in the low range of normal values. The patient was transfused with 3 units of FFP.

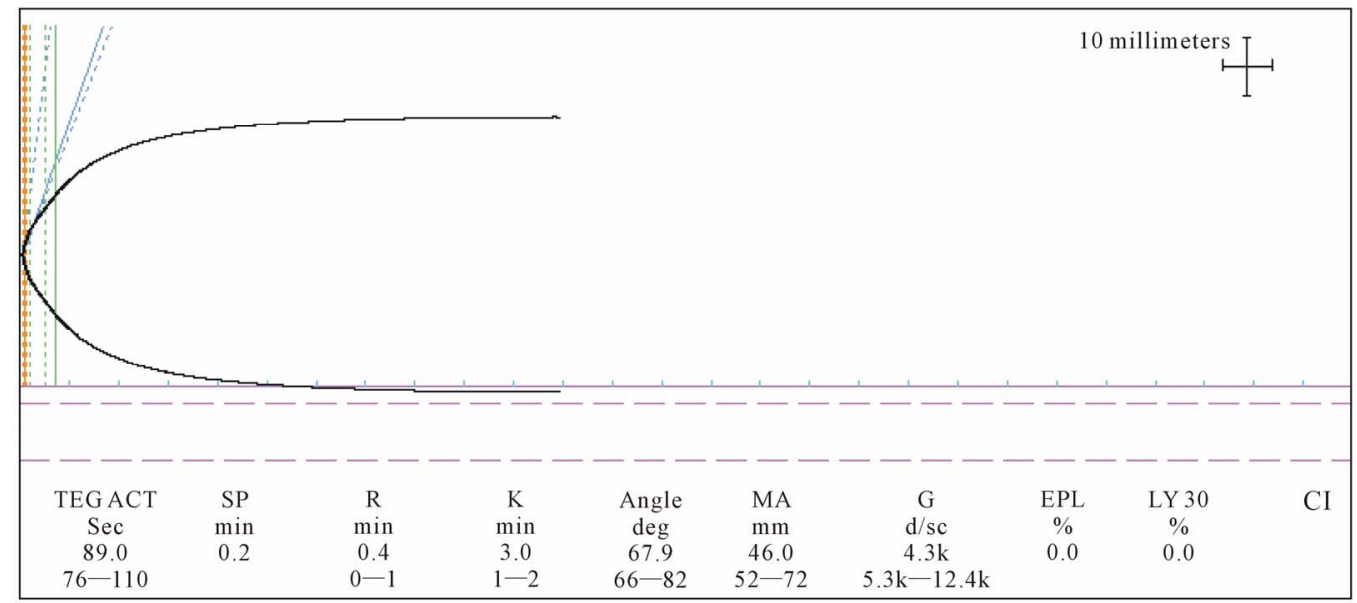

Figure 4. Reperfusion phase Rapid TEG ${ }^{\circledR}:$ MA and G values slightly below normal and a prolonged $\mathrm{K}$ time. The platelet count was 46,000 u/L. During this period he received 1 unit of SDP and 10 units of cryoprecipitate.

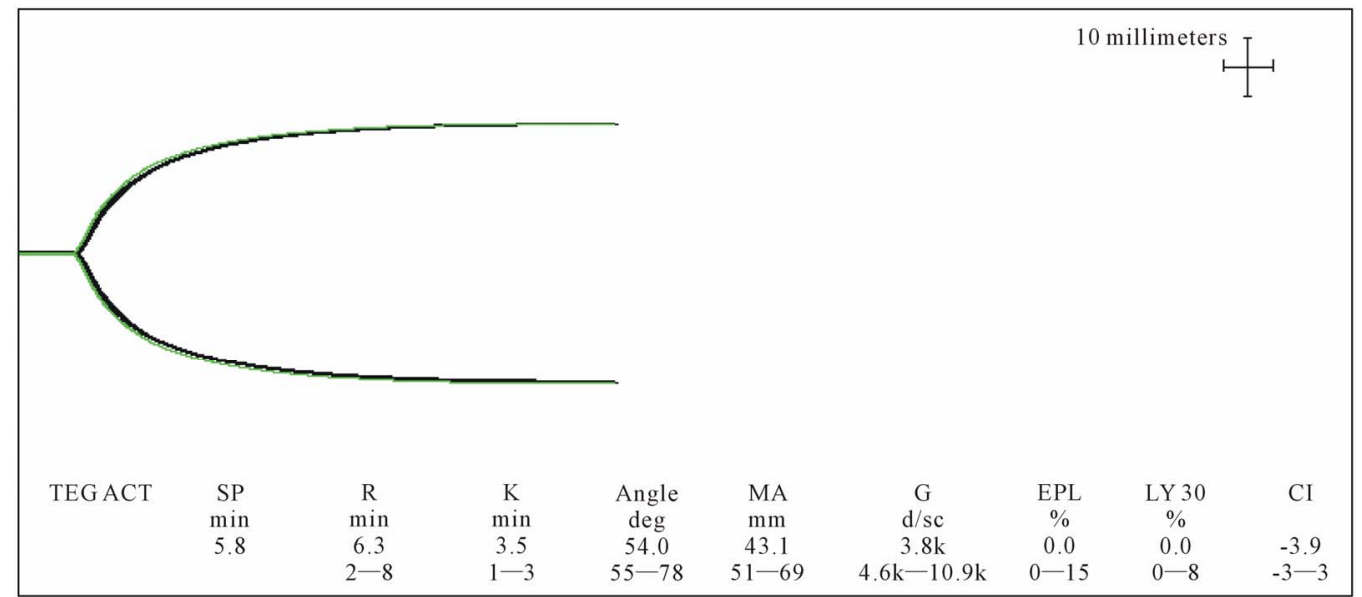

Figure 5. ICU Basic TEG ${ }^{\circledR}$. Basic TEG ${ }^{\circledR}$ is done with $340 \mu \mathrm{l}$ of whole blood and $20 \mu \mathrm{l}$ of CaCl. Then two cups are run, one of them has heparinase added to remove heparin in the sample and measure the effect of heparin. An angle of 54 indicated decreased fibrinogen. A MA of $43 \mathrm{~mm}$ (3.7 SD below the mean) indicated poor platelet function. A G value of $3.8 \mathrm{~K}$ showed reduced clot strength. 
Table 1. Summary of TEG $^{\circledR}$ values.

\begin{tabular}{|c|c|c|c|c|c|c|c|c|}
\hline TEG $^{\circledR}$ & $\begin{array}{c}\text { PRE } \\
\text { Anhepatic }\end{array}$ & $\begin{array}{c}\text { 1st } \\
\text { Anhepatic }\end{array}$ & $\begin{array}{c}\text { 2nd } \\
\text { Anhepatic }\end{array}$ & Neo-hepatic & ICU & 6 days POP & \multicolumn{2}{|c|}{ Normal values } \\
\hline Type of TEG ${ }^{\circledR}$ & Rapid TEG ${ }^{\circledR}$ & Rapid TEG $^{\circledR}$ & Rapid TEG $^{\circledR}$ & Rapid TEG ${ }^{\circledR}$ & Citrated kaolin & Citrated Kaolin & Rapid TEG ${ }^{\circledR}$ & Citrated kaolin \\
\hline TEG $^{\circledR}$ ACT & 300 & 89 & 97 & 89 & & & $76-110 \mathrm{sec}$ & \\
\hline $\mathbf{R}$ & 2.7 & 0.4 & 0.5 & 0.4 & 6.3 & 5.8 & $0-1$ min & $2-8 \min$ \\
\hline $\mathbf{K}$ & 5.2 & 7.2 & 1.8 & 3 & 3.5 & 4.2 & $1-2 \min$ & $1-3$ min \\
\hline Angle & 41.4 & 63.1 & 74.8 & 67.9 & 54 & 47.7 & 66 - 82 deg & 55 - 78 deg \\
\hline MA & 38 & 30.2 & 53.8 & 46 & 43.1 & 41.8 & $52-72$ mm & $51-69 \mathrm{~mm}$ \\
\hline G & 3.1 & 2.2 & 5.8 & 4.3 & 3.8 & 3.6 & $5.3-12.4 \mathrm{k}$ & $4.6-10.9 \mathrm{k}$ \\
\hline
\end{tabular}

ACT: Activated clotting time; $\mathbf{R}$ time: time elapsed from placement of the sample in the cup until the tracing amplitude reaches 2 mm. Marks the rate of initial fibrin formation (the burst of thrombin formation). Functionally is related to plasma clotting factors; $\mathbf{K}$ time: represents the time it takes for a fixed degree of viscoelasticity to be achieved by the forming clot. It is influenced by the activity of intrinsic clotting factors and fibrinogen. Is measured from $\mathrm{R}$ to the point at which the amplitude reaches $20 \mathrm{~mm}$; Angle: determines the rate at which the clot is formed. It is formed by the slope of the TEG tracing from the R to K values. Functionally reflects fibrinogen activity; MA is the maximal amplitude on the TEG tracing. Reflects the strength of the platelet function and can be altered by platelet abnormalities in number or in quality; G: strength of the clot with all components interacting (factors, fibrinogen, and platelets).

\section{Discussion}

Hemophilia $\mathrm{A}$ is an inherited bleeding disorder due to factor VIII deficiency. Many hemophiliacs who received clotting factors concentrates prior to the mid 1980s were infected with the hepatitis $C$ virus and a significant group of them have progressed to cirrhosis and hepatocellular carcinoma [2].

Hemophilia is inherited as an $\mathrm{X}$ linked recessive trait. The severity is classified into three categories: severe deficiency $(0 \%-1 \%$ of normal), moderate deficiency (1\% - 5\%) and mild deficiency (5\% - 25\%).

Orthotopic liver transplantation (OLT) is the only cure for end stage liver disease and hemophilia. Liver transplantation in hemophiliacs reverses hepatic failure and corrects coagulation defects but not genetic transmissibility. End stage liver disease combined with hemophilia exposes the patient to a greater risk of bleeding complications during OLT, although, these patients can be safely brought through OLT without excessive transfusion requirements, using appropriate replacement regimens [3].

TE is a method for evaluating the viscoelastic properties of blood clots from formation to its lysis. It performs a qualitative and dynamic analysis of specific blood clotting process, highlighting alterations at every single step in the coagulation cascade [1]. With TE it is possible to know if bleeding is due to failure of an adequate surgical hemostasis, to platelet dysfunction and to anomalies of coagulation proteases and their inhibitors. TE is readily available in the operating room and can provide feedback in terms of graft function after reperfusion [4]. Reports have shown that TE use during OLT can decrease blood product transfusion requirement by as much as 33\% compared with those monitored by conventional coagulation tests. Transfusion practices during OLT differ among transplant centers, and vary according to the meth- od used for monitoring coagulation, the surgeon's experience, and anesthesia management $[4,5]$.

In our case, the initial thrombelastogram is a classic example of the profound alterations seen in coagulopathy from liver disease. The Factor VIII infusion and cryoprecipitate transfused prior to surgery may have partially corrected several TE values such as the $\mathrm{K}$ time, $\mathrm{R}$ time and $\mathrm{G}$ strength.

During the anhepatic phase, the abnormalities caused by surgical bleeding of the hepatectomy were normalized by transfusion products, as evidenced by the thrombelastogram and gave the surgeon a new "baseline" where the new graft was allowed to start functioning.

During the case no factor VIII neither desmopressine were required and even the initial infusion of factor VIII did not appear to have a hypercoagulable effect.

The ICU TEG ${ }^{\circledR}$ and the TEG ${ }^{\circledR} 6$ days postoperatively revealed prolonged $\mathrm{K}$ times which are supposed to be related to the activity of intrinsic clotting factor and fibrinogen. The decreased MA values can be related in theory to persistent thrombocytopenia and to functional impairment of platelets caused by Epoprostenol infusion. Despite the abnormalities observed in the follow-up thrombelastograms, the patient had no clinical bleeding; therefore it is important to emphasize that TE during OLT is an adjunct in the patient's management but adequate surgical technique and hemostasis really determine the clinical significance of laboratory values.

In conclusion, TE was of paramount importance in the perioperative transfusion management of our hemophiliac patient undergoing OLT. Specifically, TE was helpful to avoid over-transfusion and to detect any unanticipated hypercoagulable milieu, which can be detrimental in a complex vascular surgery such as OLT. Factor VIII infusion prior to or after transplant had no pro-thrombotic 
side effects on the vascular anastomosis of the graft, as evidenced by the follow up thrombelastogram six days post transplant and normal graft function. OLT restored normal liver function and cured this patient's hemophilia.

\section{REFERENCES}

[1] E. G. Pivalizza, "Perioperative Use of Thrombelastograph $^{\circledR}$ in Patients with Inherited Bleeding Disorders,” Journal of Clinical Anesthesia, Vol. 15, No. 5, 2003, pp. 366-370. doi:10.1016/S0952-8180(03)00022-9

[2] M. Franchini, N. Nicolini and F. Capra, "Treatment of Hepatitis C in Hemophiliacs," American Journal of Hematology, Vol. 81, No. 9, 2006, pp. 696-702. doi:10.1002/ajh.20701

[3] J. Wilde, P. Teixeira, S. R. Bramhall, B. Gunson, D. Mu- timer and D. F. Mirza, "Liver Transplantation in Haemophilia,” British Journal of Haematology, Vol. 117, No. 4, 2002, pp. 952-956. doi:10.1046/j.1365-2141.2002.03528.x

[4] M. Coakley, K. Reddy, I. Mackie and S. Mallet, “Transfusion Triggers in Orthotopic Liver Transplantation: A Comparison of Thromboelastometry Analyzer, the Thromboelastogram, and Conventional Coagulation Tests," Journal of Cardiothoracic and Vascular Anesthesia, Vol. 20, No. 4, 2006, pp. 548-553. doi:10.1053/j.jvca.2006.01.016

[5] A. A. Ashrani, M. T. Reding, A. Shet, J. Osip, A. Humar, J. R. Lake, et al., "Successful Liver Transplantation in a Patient with Severe Haemophilia A and a High-Titer Factor VIII Inhibitor,” Haemophilia, Vol. 10, No. 6, 2004, pp. 735-737. 